\title{
Efficacy of Adenine in the Treatment of Leukopenia and Neutropenia Associated with an Overdose of Antipsychotics or Discontinuation of Lithium Carbonate Administration: Three Case Studies
}

\author{
Takashi Tomita ${ }^{1}$, Hidekazu Goto ${ }^{1}$, Kenji Sumiya ${ }^{2}$, Tadashi Yoshida ${ }^{3}$, Katsuya Tanaka ${ }^{4}$, Yukinao Kohda ${ }^{1,5}$ \\ Departments of ${ }^{1}$ Pharmacy, ${ }^{3}$ Internal Medicine, and ${ }^{4}$ Psychiatry, Hospital Bando, Bando, ${ }^{2}$ Faculty of Pharmacy, Iwaki Meisei University, \\ Iwaki, ${ }^{5}$ Faculty of Health Sciences, Tsukuba International University, Tsuchiura, Japan
}

\begin{abstract}
Because adenine is effective for managing cases of radiation-induced and drug-induced leukopenia, it may be effective in cases of antipsychotic-induced leukopenia and neutropenia. Here, we report our experience with patients with leukopenia and neutropenia caused by an antipsychotic overdose or discontinuation of lithium carbonate, in whom adenine administration ameliorated the white blood cell and neutrophil counts. The progress of patients suggests that adenine is effective in cases of leukopenia and neutropenia associated with lithium carbonate discontinuation and an antipsychotic overdose.
\end{abstract}

KEY WORDS: Adenine; Leukopenia; Neutropenia; Antipsychotic agents; Lithium carbonate.

\section{INTRODUCTION}

Because hematologic diseases that develop as side effects of drugs have the risk causing abrupt deterioration leading to death, sufficient caution is needed when receiving drugs. Leukopenia accounts for the majority of cases of hematologic diseases, and the frequency of granulocytopenia, an acute condition characterized by a decreased neutrophil count, is especially high. The basic treatment for drug-induced granulocytopenia in patients receiving antipsychotics is the identification and discontinuation of the responsible drug and simultaneous prevention of infection. However, in patients receiving multi-antipsychotic combination therapy, identification of the granulocytopenia-causing drug is difficult, and in some cases, discontinuation of the antipsychotic may ag-

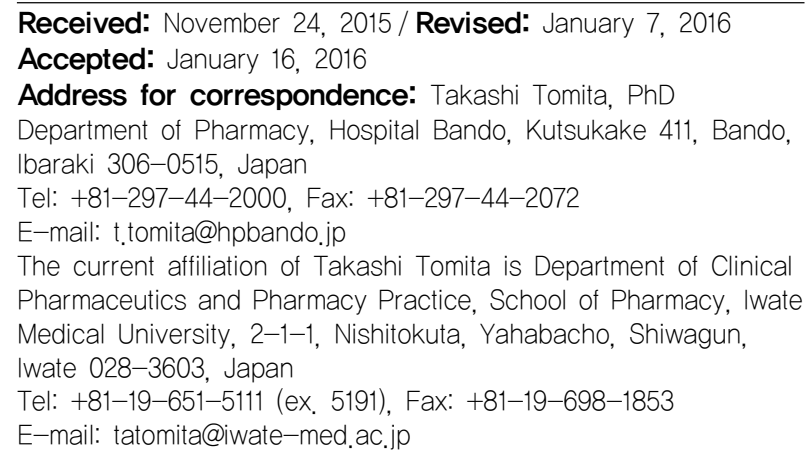

gravate psychiatric symptoms.

Because adenine is effective for managing cases of radiation-induced and drug-induced leukopenia, ${ }^{1,2)}$ it may be effective in cases of antipsychotic-induced leukopenia and neutropenia. Here, we report our experience with patients with leukopenia and neutropenia caused by an antipsychotic overdose or discontinuation of lithium carbonate, in whom adenine administration ameliorated the white blood cell (WBC) and neutrophil counts.

\section{CASE}

\section{Case 1}

A 58-year-old women with bipolar disorder, who was in a manic state and in whom adenine administration was effective in treating leukopenia associated with the discontinuation of lithium carbonate (Fig. 1).

The patient started receiving $800 \mathrm{mg}$ /day lithium carbonate at the age of 57 years and 10 months to manage manic episodes of bipolar disorder. The therapeutic range of lithium for prophylaxis is between $0.6-0.8 \mathrm{mEq} / \mathrm{L}$ and in acute treatment is $0.8-1.2 \mathrm{mEq} / \mathrm{L}$.

In hospitalized patients, WBC and neutrophil counts that are considered 'normal' range from $3,500 / \mu 1$ to $9,000 / \mu 1$ and 'normal' neutrophil counts range from $2,000 / \mu 1$ to $6,800 / \mu \mathrm{l}$.

As shown in Figure 1, at the age of 58 years and 3

(a) This is an Open-Access article distributed under the terms of the Creative Commons Attribution Non-Commercial License (http://creativecommons.org/licenses/by-nc/4.0) which permits unrestricted non-commercial use, distribution, and reproduction in any medium, provided the original work is properly cited. 

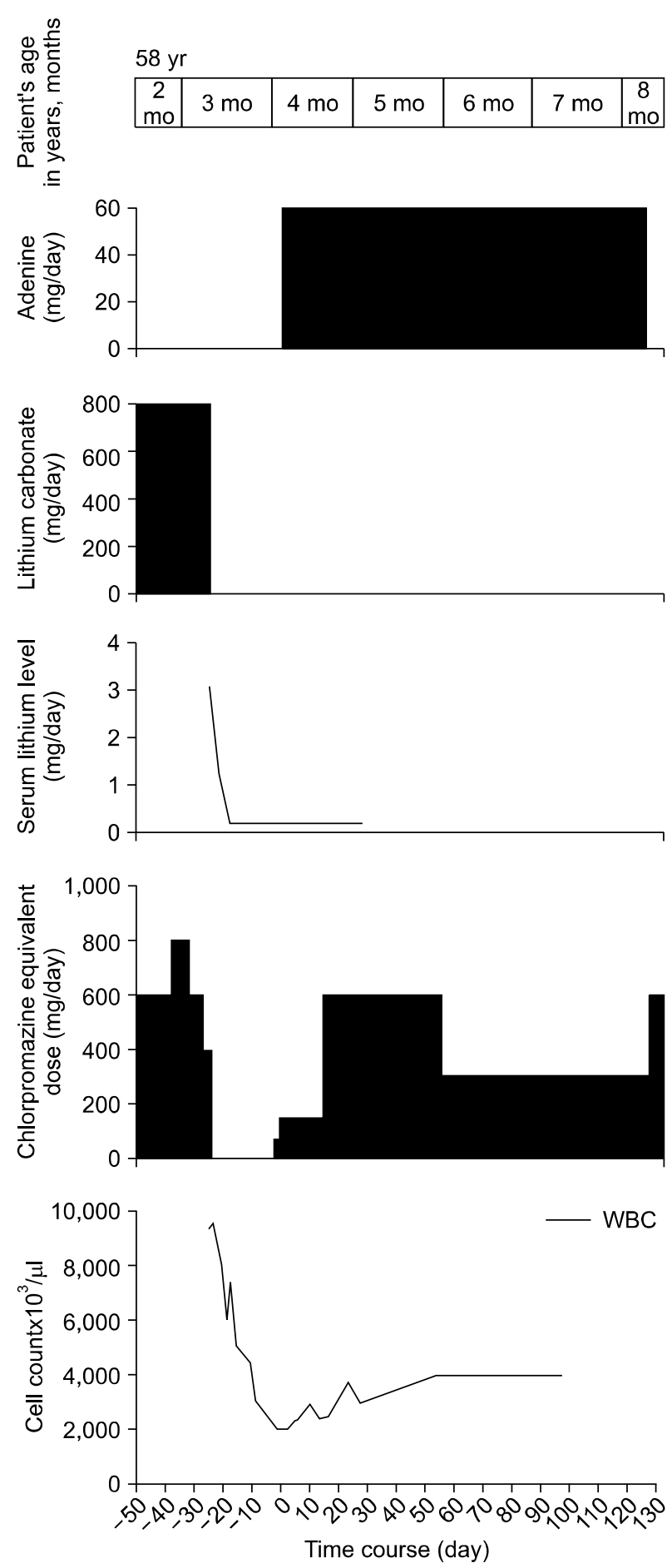

Fig. 1. Efficacy of adenine in the treatment of leukopenia associated with the discontinuation of lithium carbonate administration; patient 1. WBC, white blood cell count.

months, the patient took an overdose of lithium carbonate at home; therefore, we performed an emergency examination of serum lithium concentration. Because a blood test revealed a serum lithium concentration of $3.11 \mathrm{mEq} / \mathrm{L}$, lithium carbonate was immediately discontinued. Seven days after discontinuation, the serum lithium concentration decreased to $0.2 \mathrm{mEq} / \mathrm{L}$.

Moreover, her WBC count was $9,400 / \mu l$ at the time of lithium carbonate discontinuation, but the count decreased to 2,000/ $\mu 124$ days after discontinuation. Because the patient developed leukopenia, at the age of 58 years and 4 months, $60 \mathrm{mg} /$ day adenine treatment was initiated to prevent infection and to increase the WBC count after adenine administration, the WBC count gradually increased, and by day 23 after adenine initiation, the count increased to $3,700 / \mu 1$.

During this time period, she was treated with quetiapine (started with $25 \mathrm{mg} /$ day, then increased to $200 \mathrm{mg}$ /day), aripiprazole (12 mg/day).

\section{Case 2}

A 54-year-old man with schizophrenia in whom adenine was effective in managing leukopenia and neutropenia caused by lithium carbonate discontinuation and antipsychotic overdose (Fig. 2).

The patient has previously developed leukopenia associated with increased chlorpromazine equivalent doses (not in shown in Fig. 2). At the age of 53 years and 4 months, the chlorpromazine equivalent dose was $800 \mathrm{mg}$ and his WBC count was 4,000/ $\mu \mathrm{l}$; however, at the age of 53 years and 5 months, the chlorpromazine equivalent

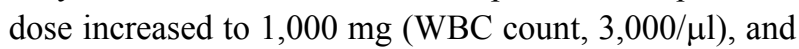
then to $1,200 \mathrm{mg}$ (WBC count, 2,900/ $\mu \mathrm{l}$ ) and 1,400 mg (WBC count, 2,500/ $\mu \mathrm{l}$ ) in the next 2 months respectively. Owing to an increase in the chlorpromazine equivalent dose, WBC count decreased from $3,000 / \mu 1$ to $2,500 / \mu 1$ (data not shown).

In spite of the risk of excessive dosing and polypharmacy, he received excessive dosing (defined as mean chlorpromazine equivalent doses above 1,000 mg) and polypharmacy of antipsychotics (combination use of different antipsychotics) owing to improve the psychiatric symptoms.

At the age of 53 years and 7 months, the WBC and neutrophil counts decreased to $1,900 / \mu 1$ and $900 / \mu 1$, respectively. Therefore, we discontinued the antipsychotic to increase the $\mathrm{WBC}$ count. Three days after discontinuation, the WBC and neutrophil counts increased to 3,700/ $\mu 1$ and $2,200 / \mu 1$, respectively. Moreover, 10 days after discontinuation, the corresponding counts were $5,600 / \mu 1$ and $3,700 / \mu l$ (data not shown).

As shown in Fig. 2, during the period from age 53 years 


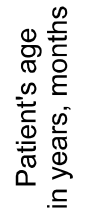

$53 \mathrm{yr}$
\begin{tabular}{|c|c|c|c|}
\hline $9 \mathrm{mo}$ & $10 \mathrm{mo}$ & $11 \mathrm{mo}$ & $0 \mathrm{mo}$ \\
\hline
\end{tabular}
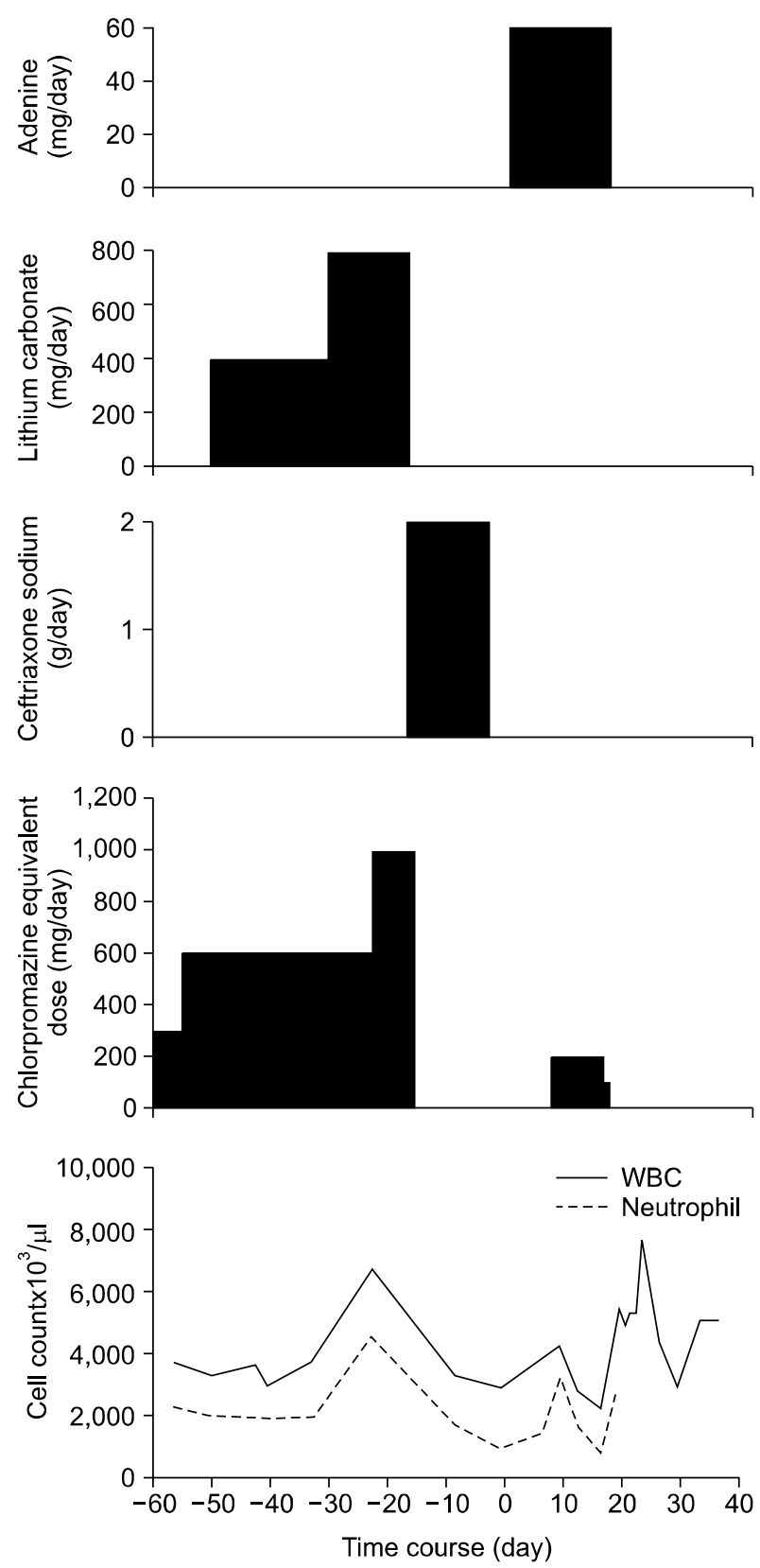

Fig. 2. Efficacy of adenine in the treatment of leukopenia and neutropenia associated with an overdose of antipsychotics and discontinuation of lithium carbonate administration; patient 2 . WBC, white blood cell count.

and 9 months to 53 years and 10 months, the equivalent dose of chlorpromazine was ranging from $300 \mathrm{mg}$ to 600 $\mathrm{mg}$, WBC count was ranging from $3,000 / \mu 1$ to $3,800 / \mu 1$, and neutrophil count was ranging from $1,900 / \mu \mathrm{l}$ to $2,300 / \mu 1$.

At age 53 years and 10 months, the chlorpromazine equivalent dose increased to $1,000 \mathrm{mg}$, but antipsychotics, including lithium carbonate $(800 \mathrm{mg} /$ day $)$, were discontinued owing to aspiration pneumonia. Owing to the development of aspiration pneumonia, WBC and neutrophil counts increased to $6,700 / \mu \mathrm{l}$ and $4,600 / \mu 1$, respectively, but following venous administration of ceftriaxone sodium ( $2 \mathrm{~g} /$ day), the corresponding counts decreased to $3,300 / \mu \mathrm{l}$ and $1,700 / \mu 1$. Moreover, 3 days after ceftriaxone discontinuation, WBC count and neutrophil count decreased to $2,900 / \mu 1$ and $900 / \mu 1$, respectively.

Because leukopenia and neutropenia developed in the patient with the elevation of chlorpromazine equivalent dose levels, at the age of 53 years and 11 months, adenine (60 mg/day) was administered. Nine days after adenine administration, WBC and neutrophil counts increased to $4,200 / \mu 1$ and $3,200 / \mu 1$, respectively. Subsequently the corresponding counts decreased to $2,200 / \mu 1$ and $800 / \mu 1$. The administration of an antipsychotic (chlorpromazine equivalent dose, $200 \mathrm{mg}$ ) 7 days after adenine administration appeared to have caused neutropenia and leukopenia.

After finishing treatment with adenine, it can take anywhere from about 20 to 30 days to increase WBC and neutrophil counts. ${ }^{3)}$ During this period, WBC and neutrophil counts decreased after an antipsychotic administration (risperidone, $2 \mathrm{mg} /$ day; chlorpromazine equivalent dose, $200 \mathrm{mg}$ ). After discontinuation of antipsychotic, the WBC and neutrophil counts increased. For the above reasons, leukopenia and neutropenia may occur, especially during this period.

To avoid aspiration pneumonia in the patient, we discontinued the oral administration of antipsychotics and adenine. Three days after discontinuation, WBC and neutrophil counts increased to $5,400 / \mu \mathrm{l}$ and $2,900 / \mu 1$, respectively.

During this time period, he was treated with aripiprazole $(10 \mathrm{mg} /$ day), aripiprazole (started with $12 \mathrm{mg} /$ day, then increased to $24 \mathrm{mg} /$ day), risperidone (started with 1 $\mathrm{mg} /$ day, then increased to $2 \mathrm{mg}$ /day).

\section{Case 3}

A 62-year-old woman with schizophrenia in whom adenine was effective in managing leukopenia and neutropenia caused by an antipsychotic overdose (Fig. 3).

From age 61 years and 1 month to 61 years and 9 months (not in shown in Fig. 3), the chlorpromazine equivalent dose was ranging from $3,000 \mathrm{mg}$ to $1,350 \mathrm{mg}$, 

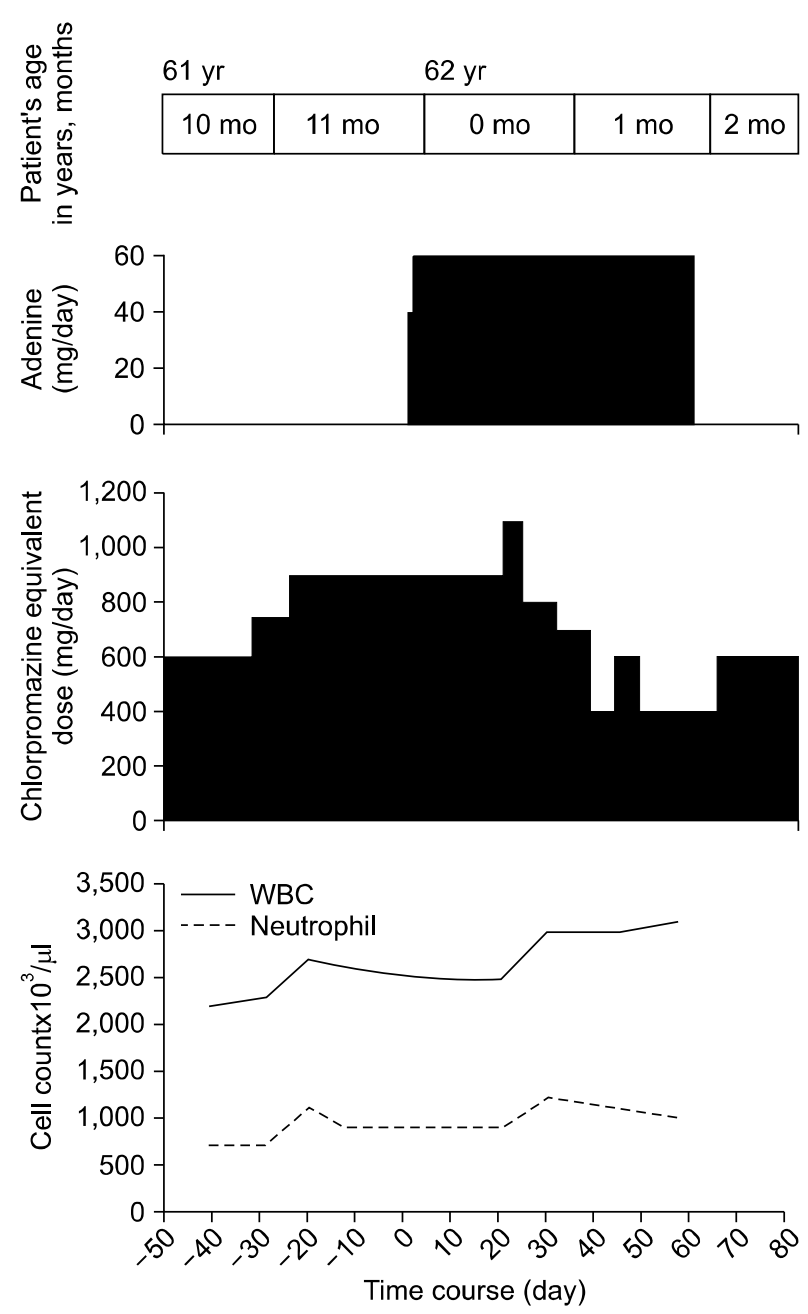

Fig. 3. Efficacy of adenine in the treatment of leukopenia and neutropenia associated with an overdose of antipsychotics; patient 3. WBC, white blood cell count.

and $\mathrm{WBC}$ and neutrophil counts were ranging from $2,600 / \mu 1$ to $2,200 / \mu 1$ and $1,000 / \mu 1$ to $700 / \mu 1$, respectively. In spite of the risk of excessing dosing and polypharmacy, he received excessive dosing (defined as mean chlorpromazine equivalent doses above $1,000 \mathrm{mg}$ ) and polypharmacy of antipsychotics (combination use of different antipsychotics) owing to improve the psychiatric symptoms. Because leukopenia and neutropenia were not ameliorated after approximately 8 months, we took countermeasures against infection. Because the chlorpromazine equivalent dose increased from $1,350 \mathrm{mg}$ to 3,000 $\mathrm{mg}$, leukopenia and neutropenia appeared to have been caused an antipsychotic overdose (data not shown).

To increase the WBC and neutrophil counts, the chlorpromazine equivalent dose was decreased in stages from $3,000 \mathrm{mg}$ to $1,350 \mathrm{mg}$, but WBC and neutrophil counts did not increase (data not shown).

From age 61 years and 9 months to 61 years and 10 months, WBC and neutrophil counts decreased from $2,600 / \mu 1$ to $2,200 / \mu 1$ and from $1,000 / \mu 1$ to $700 / \mu 1$. Hence, the chlorpromazine equivalent dose was reduced from $1,350 \mathrm{mg}$ to $600 \mathrm{mg}$. However, WBC and neutrophil counts did not increase. Therefore, at the age of 61 years and 11 months (as shown in Fig. 3), we initiated $60 \mathrm{mg}$ /day adenine administration to increase WBC and neutrophil counts (the chlorpromazine equivalent dose was increased from $750 \mathrm{mg}$ to $900 \mathrm{mg}$ ).

After the initiation of adenine administration, it was therapeutically necessary to temporarily increase the chlorpromazine equivalent dose to $1,100 \mathrm{mg}$ (at the age of 62 years and 0 month), and the WBC and neutrophil counts gradually increased with no subsequent decrease. Thirty days after the initiation of adenine administration, neutrophil count increased to $1,200 / \mu$ l. This is mostly due to insufficient administration of adenine. It is necessary to administer adenine until recovery of neutropenia. Fifty seven days after adenine initiation, WBC count increased to $3,100 / \mu 1$.

During this time period, he was treated with bromperidol (started with $3 \mathrm{mg} /$ day, then increased to $18 \mathrm{mg} /$ day), aripiprazole (started with $6 \mathrm{mg}$ /day, then increased to 24 $\mathrm{mg}$ /day), olanzapine (started with $5 \mathrm{mg} /$ day, then increased to $10 \mathrm{mg} /$ day).

\section{DISCUSSION}

We administered adenine to manage leukopenia (WBC count, $<3,000 / \mu \mathrm{l}$ ) and neutropenia (neutrophil count, $<1,500 / \mu \mathrm{l}$ ) while confirming WBC and neutrophil counts through appropriate hematological testing. In our patients, there were no adverse events with the administration of adenine.

Adenine was administered until the WBC becomes to be 3,000/ $\mu 1$. Because the patient 1 was discharged from hospital, she was administered with adenine for about 4 months. In hospitalized patients, WBC and neutrophil counts that are considered 'normal' range from $3,500 / \mu$ lo $9,000 / \mu l$ and 'normal' neutrophil counts range from $2,000 / \mu 1$ to $6,800 / \mu 1$. Although the details of the mechanism underlying adenine's efficacy against radiation exposure and leukopenia are unclear, adenine is considered the active substance involved in leukocyte equilibrium because it is absorbed by bone marrow RNA/DNA and used in nucleic acid synthesis.

Few previous studies have examined the efficacy of ad- 
enine formulations in managing neutropenia associated with the administration of antipsychotics, including clozapine, which is known to lead to a high frequency of drug-induced neutropenia. ${ }^{4)}$ The lack of reports examining adenine's efficacy may be attributable to the fact that adenine formulations are only sold in three countries: Japan, Spain, and France. However, recently, Takeuchi et $a l .^{4)}$ have reported adenine's efficacy in cases of clozapine-associated neutropenia. Increased WBC and neutrophil counts have been reported in patients with bipolar disorder who received lithium carbonate. ${ }^{5-9)}$ In patients 1 and 2, the increased WBC and neutrophil counts associated with lithium carbonate appeared to decrease owing to lithium carbonate discontinuation. Our results suggest that adenine may be effective in cases of leukopenia and neutropenia due to lithium carbonate discontinuation.

Leukopenia and neutropenia appeared develop in patient 2 owing to lithium carbonate discontinuation and an antipsychotic overdose. The progress of patient 2 suggests that adenine is effective in cases of leukopenia and neutropenia associated with lithium carbonate discontinuation and in cases of leukopenia and neutropenia caused by an antipsychotic overdose.

Administration of antipsychotics was continued in patient 3 in whom neutropenia had been caused by an antipsychotic overdose, but neutrophil and WBC counts increased after administration of an adenine formulation. Because antipsychotics could be continued in this patient, psychiatric symptoms did not worsen.

Because of the high frequency of multi-antipsychotic combination therapy application in Japan, there is a great need in the field of psychiatry to examine adenine's effi- cacy in managing leukopenia and neutropenia. Adenine is an effective treatment, especially for patients receiving multi-antipsychotic combination therapy in whom the drug causing leukopenia or neutropenia cannot be identified, since it would allowing for identification of the drug. Moreover, it is effective in patients who are at a risk of exacerbation of psychiatric symptoms due to discontinuation of the drug causing leukopenia or neutropenia, allowing a switch to be made to a safer drug.

\section{REFERENCES}

1. Lecoq R. Anti-agranulocytic vitamin B4. Int Z Vitaminforsch Beih 1957;27:291-300. French.

2. Paolino W, Vercellino E. Effects of adenine on blood disease due to benzene poisoning. Panminerva Med 1960;2:5-8.

3. Fukui H, Suwa T, Yamamura S, Murai T. Successful treatment of neuroleptic-induced neutropenia by adenine. Jpn J Psychiatr Treat. 2001;16:1085-1088.

4. Takeuchi I, Kishi T, Hanya M, Uno J, Fujita K, Kamei H. Effect of adenine on clozapine-induced neutropenia in patients with schizophrenia: A preliminary study. Clin Psychopharmacol Neurosci 2015;13:157-162.

5. Mayfield D, Brown RG. The clinical laboratory and electroencephalographic effects of lithium. J Psychiatr Res 1966;4:207-219.

6. Murphy DL, Goodwin FK, Bunney WE Jr. Leukocytosis during lithium treatment. Am J Psychiatry 1971;127:15591561.

7. Shopsin B, Friedmann R, Gershon S. Lithium and leukocytosis. Clin Pharmacol Ther 1971;12:923-928.

8. Bille PE, Jensen MK, Kaalund Jensen JP, Poulsen JC. Studies on the haematologic and cytogenetic effect of lithium. Acta Med Scand 1975;198:281-286.

9. Watanabe S, Taguchi K, Nakashima Y, Ebara T, Iguchi K. Leukocytosis during lithium treatment and its correlation to serum lithium level. Folia Psychiatr Neurol Jpn 1974; 28:161-165. 